\title{
Determination of intrinsic switching field distributions in perpendicular recording media: numerical study of the $\Delta H(M, \Delta M)$ method
}

\author{
Yang Liu and Karin A. Dahmen \\ Department of Physics, University of Illinois at Urbana-Champaign, Urbana, IL 61801, USA \\ A. Berger \\ San Jose Research Center, Hitachi Global Storage Technologies, San Jose, CA 95135, USA
}

(Dated: August 28, 2021)

\begin{abstract}
We present a numerical study of the $\Delta H(M, \Delta M)$ method and its ability to accurately determine intrinsic switching field distributions in interacting granular magnetic materials such as perpendicular recording media. In particular, we study how this methodology fails for large ferromagnetic inter-granular interactions, at which point the associated strongly correlated magnetization reversal cannot be properly represented by the mean-field approximation, upon which the $\Delta H(M, \Delta M)$ method is based. In this study, we use a 2-dimensional array of symmetric hysterons that have an intrinsic switching field distribution of standard deviation $\sigma$ and ferromagnetic nearest-neighbor interactions $J$. We find the $\Delta H(M, \Delta M)$ method to be very accurate for small $J / \sigma$ values, while substantial errors develop once the effective exchange field becomes comparable with $\sigma$, corroborating earlier results from micromagnetic simulations. We furthermore demonstrate that this failure is correlated with deviations from data set redundancy, which is a key property of the mean-field approximation. Thus, the $\Delta H(M, \Delta M)$ method fails in a well defined and quantifiable manner that can be easily assessed from the data sets alone.

PACS numbers: 75.50.Ss, 75.60.Ej, 75.75.+a
\end{abstract}

\section{INTRODUCTION}

One of the key challenges in advancing the nanotechnology of magnetic recording is the optimization of recording media and its physical properties 1 . This challenge is particularly demanding because magnetic recording is by its nature a local process. Thus, it is not so much the average physical properties that are crucial, but the distributions of such properties that determine continued technological advancement and success ${ }^{1}$. In general, it is important to devise recording media structures that have very homogeneous properties on recording relevant length scales, so that a position independent physical description of all properties and magnetization processes is appropriate. However, this can only be achieved to a limited degree and it is therefore essential to have exact knowledge of the corresponding parameter distributions. One of the most crucial properties is the intrinsic switching-field distribution $D\left(H_{\mathrm{S}}\right)$ of the media grains because it defines the recording quality of a media layer in both magnetic stability and the achievable recording density $^{2}$. Hereby, one has to realize that it is not the macroscopic switching field distribution $D_{\mathrm{m}}\left(H_{\mathrm{S}}\right)$ in a uniformly applied field that is relevant, but the local distribution $D\left(H_{\mathrm{S}}\right)$ of switching fields in a recording process, which takes place in a narrowly defined field geometry. The difference between $D_{\mathrm{m}}\left(H_{\mathrm{S}}\right)$ and $D\left(H_{\mathrm{S}}\right)$ is caused by the inter-granular interactions between the media grains. In particular, for perpendicular media the dipolar interaction is large and dominates the behavior of hysteresis loops $M(H)$. Thus, the knowledge of $D_{\mathrm{m}}\left(H_{\mathrm{S}}\right)$, which can be derived from the slope of $M(H)$, is insufficient for recording performance predictions.
Over the years, several methodologies have been developed to determine $D\left(H_{\mathrm{S}}\right)$ with varying success $3,4,5,6,7,8,9$. Most of these methods apply a measurement scheme, in which magnetization reversal of media grains is probed starting from different magnetization states to allow for a variation of the grain-to-grain interaction under measurement conditions. Such procedures should then in principle and under certain limiting conditions allow for a separation of the intrinsic switching field distribution and the inter-granular interactions.

In this paper, we study the reliability of the recently developed $\Delta H(M, \Delta M)$ method, which has been used successfully in analyzing progress in recording media fabrication ${ }^{7,8}$. The method itself is a generalization of an earlier measurement technique, the $\Delta H_{\mathrm{C}}$ method $^{3}$, but has the advantage that it allows the determination of the entire $D\left(H_{\mathrm{S}}\right)$ distribution and not just a single characteristic parameter. Furthermore, it enables oversampling, which makes consistency checks feasible and gives one the opportunity to quantify the confidence level of measurement results. In contrast to the also quite robust method developed by van de Veerdonk et al. $\stackrel{5,6}{\text {, }}$, it does not rely on a specific distribution form or the rather limiting assumption that interactions can be removed from the problem by a simple de-shearing of the major hysteresis loop. Recent data indicate that this very assumption appears to be an overly simplistic view of inter-granular interactions ${ }^{8}$. Another method that has recently gained certain popularity is the FORC-method ${ }^{4.9}$, which is very similar to the measurement of Preisach distributions ${ }^{10}$. However, this technique cannot really be compared to the previously mentioned methods, because it represents for the most part a data transformation tool and does 
not appear to allow a self-consistent way of extracting microscopic information such as $D\left(H_{\mathrm{s}}\right)$, because all interactions are removed from the model in an ad hoc fashion simply by definition $\underline{4}, \underline{9}$.

Here, we present a numerical study of the $\Delta H(M, \Delta M)$ method at zero temperature. We show that even though this method approximates the inter-granular interactions on the mean-field level, it can predict its own reliability correctly. The paper is organized as follows. In Sec. II] we give a brief introduction to the $\Delta H(M, \Delta M)$ method. In Sec. III, we discuss the model used to numerically calculate the magnetization curves $M(H)$. In Sec. IV] we introduce reliability measures to quantify the failure of the $\Delta H(M, \Delta M)$ method. In Sec. V], we present and analyze our numerical results and a summary is given in Sec. VI.

\section{II. $\Delta H(M, \Delta M)$ METHOD}

The $\Delta H(M, \Delta M)$ method assumes that the effective field at each grain can be written as $H_{\text {eff }}=$ $H(M)+H_{\text {int }}(M)$ with $H(M)$ being the external field and $H_{\text {int }}(M)$ the volume-averaged interaction field at magnetization $M$. Assuming that $M$ is normalized to its saturation value $M_{\mathrm{S}}$, the upper branch of the major hysteresis loop driven by the major loop external field $H$, and the recoil curve starting at $M_{\mathrm{rev}}=1-\Delta M$ driven by the recoil curve external field $H_{\mathrm{r}}$ are given as:

$$
\begin{aligned}
& M=1-2 \int_{-\infty}^{-\left[H(M)+H_{\mathrm{int}}(M)\right]} D\left(H_{\mathrm{S}}\right) \mathrm{d} H_{\mathrm{S}} \\
& M=1-\Delta M-2 \int_{-\infty}^{-\left[H_{\mathrm{r}}(M)+H_{\mathrm{int}}(M)\right]} D\left(H_{\mathrm{S}}\right) \mathrm{d} H_{\mathrm{S}}
\end{aligned}
$$

respectively, i.e. field integrals over the intrinsic switching field distribution $D\left(H_{\mathrm{S}}\right)$. Defining an integral function $I(x)=\int_{-\infty}^{x} D\left(H_{\mathrm{S}}\right) \mathrm{d} H_{\mathrm{S}}$, one finds

$$
\begin{aligned}
& H(M)=-I^{-1}\left(\frac{1-M}{2}\right)-H_{\mathrm{int}}(M) \\
& H_{\mathrm{r}}(M)=-I^{-1}\left(\frac{1-M-\Delta M}{2}\right)-H_{\mathrm{int}}(M)
\end{aligned}
$$

with $I^{-1}$ being the inverse of the integral function. Therefore, one can derive

$$
\begin{aligned}
\Delta H(M, \Delta M) & \equiv H_{\mathrm{r}}(M)-H(M) \\
& =I^{-1}\left(\frac{1-M}{2}\right)-I^{-1}\left(\frac{1-M-\Delta M}{2}\right)
\end{aligned}
$$

as a closed functional form for the field axis distance $\Delta H$ between major and recoil curves, a quantity that is illustrated in Fig. 3. Important is hereby that within the framework of this approach $\Delta H$ is independent from the

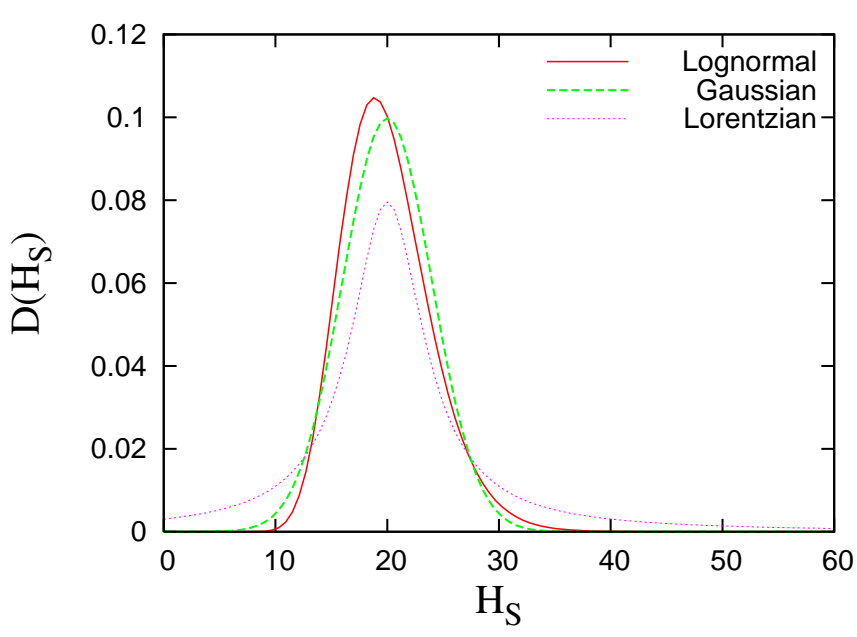

FIG. 1: Gaussian, Lorentzian and Lognormal $D\left(H_{\mathrm{S}}\right)$ distributions with the same disorder parameter $\sigma$. For both Gaussian and Lognormal distribution, the standard deviation is set to be 4.0 and the mean value 20.0. For the Lorentzian distribution, we set the width at half-maximum to be 4.0 and the center of the distribution to be 20.0 .

grain interaction, which allows for a direct experimental access to $D\left(H_{\mathrm{S}}\right)$. For certain parameterized distribution functions, one can derive analytic expressions for $\Delta H$. For example, for the Gaussian, Lorentzian and Lognormal $D\left(H_{\mathrm{S}}\right)$ distributions (see Fig. 1),

$$
\begin{aligned}
D_{\mathrm{G}}\left(H_{\mathrm{S}}\right) & =\frac{1}{\sqrt{2 \pi} \sigma} \exp \left[-\frac{\left(H_{\mathrm{S}}-h_{0}\right)^{2}}{2 \sigma^{2}}\right] \\
D_{\mathrm{L}}\left(H_{\mathrm{S}}\right) & =\frac{2 w}{\pi} \frac{1}{w^{2}+4\left(H_{\mathrm{S}}-h_{0}\right)^{2}} \\
D_{\mathrm{LN}}\left(H_{\mathrm{S}}\right) & =\frac{1}{\sqrt{2 \pi} \tilde{\sigma} H_{\mathrm{S}}} \exp \left[-\frac{\left(\log H_{\mathrm{S}}-\tilde{\mu}\right)^{2}}{2 \tilde{\sigma}^{2}}\right]
\end{aligned}
$$

we find

$$
\begin{aligned}
\Delta H_{\mathrm{G}}(M, \Delta M) & =\sqrt{2} \sigma\left(\operatorname{erf}^{-1}(M+\Delta M)-\operatorname{erf}^{-1}(M)\right) \\
\Delta H_{\mathrm{L}}(M, \Delta M)= & \frac{w}{2}\left\{\tan \left[\frac{\pi}{2}(M+\Delta M)\right]-\tan \left[\frac{\pi}{2} M\right]\right\} \\
\Delta H_{\mathrm{LN}}(M, \Delta M)= & \exp \left[\tilde{\mu}-\sqrt{2} \tilde{\sigma} \operatorname{erf}^{-1}(M)\right] \\
& -\exp \left[\tilde{\mu}-\sqrt{2} \tilde{\sigma} \operatorname{erf}^{-1}(M+\Delta M)\right]
\end{aligned}
$$

Results for the Gaussian and Lorentzian distributions were reported previously ${ }^{7,8}$. Here, we introduce the disorder parameter $\sigma$ for a general $D\left(H_{\mathrm{S}}\right)$ distribution. $\sigma$ is defined to be the standard deviation of a given distribution, such as Gaussian and Lognormal. However, for the Lorentzian distribution neither variance nor higher moments are defined, so that we need to quantify the disorder level in another form. For the Lorentzian, we define 
the disorder parameter to be the distribution width at the half-maximum and the mean to be the center of the distribution. For the distributions given by Eq. 4a, 4b and $4 \mathrm{c}$, we then have the disorder parameter: $\sigma^{\mathrm{G}}=\sigma$, $\sigma^{\mathrm{L}}=w, \sigma^{\mathrm{LN}}=e^{\tilde{\mu}+\tilde{\sigma}^{2} / 2}\left(e^{\tilde{\sigma}^{2}}-1\right)^{1 / 2}$ and the mean value $h_{0}^{\mathrm{G}}=h_{0}, h_{0}^{\mathrm{L}}=h_{0}, h_{0}^{\mathrm{LN}}=e^{\tilde{\mu}+\tilde{\sigma}^{2} / 2}$.

By making a least-squares fit of the $\Delta H(M, \Delta M)$ curves to the above formulas, one can extract the key features of $D\left(H_{\mathrm{S}}\right)$. Note that both $\Delta H_{\mathrm{G}}(M, \Delta M)$ and $\Delta H_{\mathrm{L}}(M, \Delta M)$ have no $h_{0}$ dependence. But for the Lognormal distribution, $\Delta H_{\mathrm{LN}}(M, \Delta M)$ depends on both $\tilde{\mu}$ and $\tilde{\sigma}$, and therefore it has both $\sigma$ and $h_{0}$ dependencies.

It is easy to prove that simply shifting a general distribution will not alter the $\Delta H$ data. For this, we consider the case of a general distribution $D\left(H_{\mathrm{S}}\right)$ : if we shift it towards right by an amount $H_{0}$, then the integral function $\tilde{I}(x)$ of the new distribution $\tilde{D}\left(H_{\mathrm{S}}\right)=D\left(H_{\mathrm{S}}-H_{0}\right)$ is given by $\tilde{I}(x)=I\left(x-H_{0}\right)$ and its inverse $\tilde{I}^{-1}(y)=$ $H_{0}+I^{-1}(y)$. It is then clear that this shift will not change the $\Delta H(M, \Delta M)$ formula at all, since the $H_{0}$ terms will cancel according to Eq. 3 ,

Also, we find that both $\Delta H_{\mathrm{G}}(M, \Delta M)$ and $\Delta H_{\mathrm{L}}(M, \Delta M)$ are symmetric with respect to $M=-\Delta M / 2$ while $\Delta H_{\mathrm{LN}}(M, \Delta M)$ is not symmetric. This is consistent with the original distribution: Both $D_{\mathrm{G}}\left(H_{\mathrm{S}}\right)$ and $D_{\mathrm{L}}\left(H_{\mathrm{S}}\right)$ are symmetric around $h_{0}$ while $D_{\mathrm{LN}}\left(H_{\mathrm{S}}\right)$ is not. Actually, for any $D\left(H_{\mathrm{S}}\right)$ distribution being symmetric about $h_{0}$, one finds that $I\left(h_{0}+x\right)=1-I\left(h_{0}-x\right)$ and $I^{-1}(1 / 2+y)=-I^{-1}(1 / 2-y)$, from which it is easy to prove that $\Delta H(M, \Delta M)$ is symmetric about $M=-\Delta M / 2$, i.e.

$$
\Delta H\left(-\frac{\Delta M}{2}+M^{\prime}, \Delta M\right)=\Delta H\left(-\frac{\Delta M}{2}-M^{\prime}, \Delta M\right)
$$

\section{HYSTERON MODEL}

For our numerical studies, we model each grain as a hysteron, which is the simplest mathematical construction for the description of a hysteretic system. Each hysteron will generate a rectangular hysteresis loop in an applied field $H$ as shown in Fig. 2 We assume the transition of each hysteron is infinitely sharp and it has no additional field dependence, such as finite susceptibility, for instance. The half-width of the rectangular hysteresis loop is referred to as the intrinsic switching field of the hysteron, which is a well defined property of each individual hysteron.

We also assume that the hysteron switching fields are symmetric around zero field, i.e. there is no bias. A hysteron with zero bias is called a symmetric hysteron and is consistent with the time reversal symmetry that ferromagnets exhibit $\underline{11}$. These hysteron properties can be regarded as fairly good representation of perpendicular media grains, because they have relatively

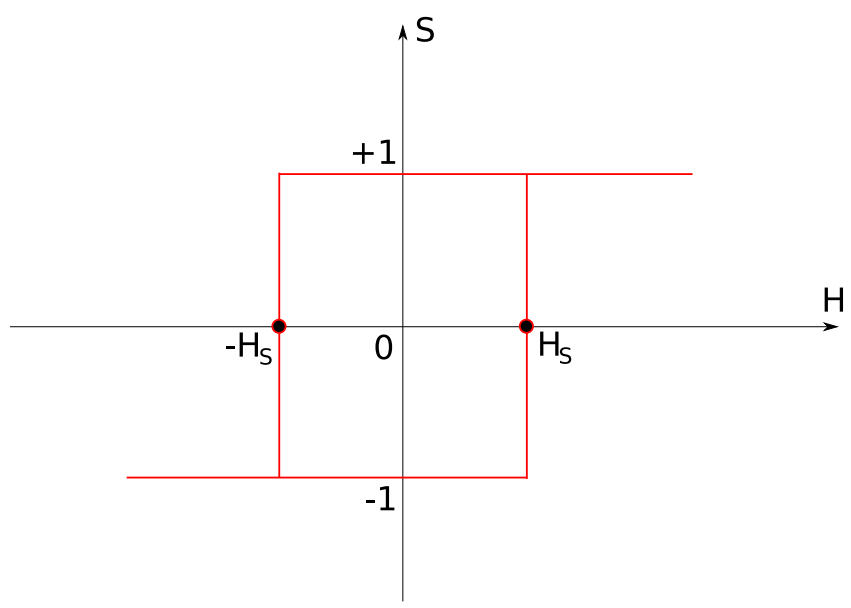

FIG. 2: A symmetric hysteron $S$ with intrinsic switching field $H_{\mathrm{S}}$.

high magnetic anisotropy and the applied field in typical characterization measurements is applied along the easy axis $3,4,5,6,7,8,9$.

We further assume that there is no time structure to the hysteron switch itself. This should be an appropriate picture as long as one considers field change rates that are much slower than single grain reversal times. Given that such reversal times are typically of the order of several hundred pico seconds $\frac{12}{2}$, this condition is generally fulfilled in typical measurement setups 13 .

In our model the ferromagnetic layer system is then represented by a simple square lattice of symmetric hysterons with periodic boundary conditions. Note that a square lattice is not necessarily a very good approximation of actual media structures, in which grains typically have coordination numbers of 5 or 6 . However, we neglect this detail of actual media structures for reasons of simplicity and furthermore assume that deviations from a more realistic grain structure are not fundamentally altering the overall significance of our study. Under the assumptions that hysterons $\left(S_{i}= \pm 1\right)$ have an intrinsic switching field distribution $D\left(H_{\mathrm{S}_{i}}\right)$ with $H_{\mathrm{S}_{i}}>0$, interact ferromagnetically with their nearest neighbors with strength $J$ and experience a uniform external field $H$, the Hamiltonian of the system can be written as

$$
\mathcal{H}=-\sum_{<i, j>} J S_{i} S_{j}-\sum_{i}\left(H+\operatorname{sgn}\left(S_{i}\right) H_{\mathrm{S}_{i}}\right) S_{i}
$$

We denote this as the random switching field hysteron model (RSFHM). Note that the RSFHM is very similar to the random field Ising model (RFIM):

$$
\mathcal{H}=-\sum_{<i, j>} J s_{i} s_{j}-\sum_{i}\left(H+h_{i}\right) s_{i}
$$

where the spins $s_{i}= \pm 1$ interact ferromagnetically with their nearest neighbors with strength $J$ and experience 
a uniform external field $H$ and a local quenched field $h_{i}$. A simple mapping: $h_{i} \leftrightarrow \operatorname{sgn}\left(S_{i}\right) H_{\mathrm{S} i}$ enables us to calculate $M(H)$ curves of symmetric hysterons with the developed algorithms used for previous RFIM computational work ${ }^{14,15}$. Details of the close relation between these models are described in Appendix A

In this paper, we evaluate different types of $D\left(H_{\mathrm{S}}\right)$ to study the reliability of the $\Delta H(M, \Delta M)$ method. In our simulation, we set the ferromagnetic nearest-neighbor coupling strength $J=1$ and tune the disorder parameter $\sigma$. Since $J=1$, tuning $\sigma$ is equivalent to tuning $\sigma / J$. In this sense, a small (big) $\sigma$ corresponds to strong (weak) nearest-neighbor interactions of hysterons. We calculate the $M(H)$ curves, both major hysteresis loop and recoil curves, for system sizes up to $1000^{2}$ and $\sigma$ values from 1.6 to 1000 . Note that we are ignoring dipolar interactions in our model (Eq. 7), even though we know that they are substantial in real structures. But a previous micromagnetic study demonstrated that the dipolar interactions can very well be treated within the mean-field $\Delta H(M, \Delta M)$ method and do not cause any significant precision problems, while exchange interactions $\operatorname{did} \underline{8}$. Thus, we focus here on the effect of intergranular exchange interactions only, because they represent the much more serious problem for the reliability of the $\Delta H(M, \Delta M)$ method. Also, we are acutely aware of the fact that the assumption of a uniform exchange coupling constant $J$ in Eq. 7 is a substantial simplification of the problem, if one compares our model to real materials structures. However, a lateral varying $J$ would be an extension of the present model and will be the topic of future work.

\section{RELIABILITY MEASURES}

In numerical test simulations of characterization methods, one can introduce a simple type of reliability measure, namely the deviation of the retrieved fit parameters from the exact solution given by the input parameters of the simulation. However, for experimental data such a reliability measure is generally not available simply because the materials parameter are not known, but are actually what one wants to extract by means of this very experimental method. Therefore, in a typical experimental situation one can only guess the overall measurement reliability by identifying parameter ranges, in which approximate methods are expected to work with a certain level of precision, which may or may not be clearly identifiable. One broadly used method to identify the reliability of extracted materials parameter is the similarity, with which a fit that is based on a certain physical model can reproduce the experimental data.

As we will demonstrate in the following, we encounter an unusually good situation for the $\Delta H(M, \Delta M)$ method, in that a consistency test of the data set alone can be used to validate the applicability of the method and estimate its overall reliability. We will furthermore show that this consistency test does not depend on any specific distribution shape but just the mean-field approximation in general, i.e. the underlying assumption of the $\Delta H(M, \Delta M)$ method.

In this section, we discuss the reliability measures of the $\Delta H(M, \Delta M)$ method for an arbitrary type of $D\left(H_{\mathrm{S}}\right)$. The reliability is characterized by two types of measures: (1) conventional quality measures for numerical fits such as the percentage difference between the fitting and the actual parameter $\left(P_{\mathrm{d}}\right)$ and the square of the multiple correlation coefficient $\left(R^{2}\right)$; (2) the average deviation from redundancy measure $(r)$, which represents the above mentioned self-consistency test.

\section{A. Fit Quality}

In simulations, one knows the input parameters, in our case the input $D\left(H_{\mathrm{S}}\right)$ distribution, exactly. Furthermore, we have also derived $\Delta H(M, \Delta M)$ formulas for certain specific $D\left(H_{\mathrm{S}}\right)$ distributions as shown in Sec. III Thus, by fitting these analytical formulas to the $\Delta H(M, \Delta M)$ curves obtained from our 2D-RSFHM hysteresis loop simulations, we can get an estimate of the input $D\left(H_{\mathrm{S}}\right)$ distribution. Obviously, if the input $D\left(H_{\mathrm{S}}\right)$ is recovered by the fitting procedure with high accuracy, then the $\Delta H(M, \Delta M)$ method works. To quantify the reliability of the $\Delta H(M, \Delta M)$ method, we introduce the following fit quality measures. The most important fit quality measure, denoted as $P_{\mathrm{d}}$, is the percentage difference between the parameters obtained from a least-square fit and the actual input parameters into our simulation. It describes how well the $\Delta H(M, \Delta M)$ method can indeed retrieve the information sought. Focusing on the disorder parameter of $D\left(H_{\mathrm{S}}\right)$ as the most crucial fit parameter, we define

$$
P_{\mathrm{d}}=\left(\sigma_{\mathrm{f}}-\sigma\right) / \sigma
$$

Here $\sigma_{\mathrm{f}}$ is the fit value of the $D\left(H_{\mathrm{S}}\right)$ distribution disorder parameter as defined in Sec. III, while $\sigma$ is the input value of the same parameter.

Another fit quality measure that can be utilized here is the square of the multiple correlation coefficient $R^{2}$, which measures how successful a fit and fit function is in explaining the data 16 . It is defined as

$$
R^{2}=1-\frac{\sum_{i}\left(Y_{i}-\hat{Y}_{i}\right)^{2}}{\sum_{i}\left(Y_{i}-\bar{Y}\right)^{2}}
$$

$Y_{i}$ and $\hat{Y}_{i}$ are hereby the simulation result and the fit function value for $\Delta H(M, \Delta M)$ at a given data point $\left(M_{i}, \Delta M_{i}\right)$, respectively. $\bar{Y}$ is the average value of $Y_{i}$. According to the definition of $R^{2}$, we know that as $R^{2}$ approaches 1 , the fit is a better and better representation of the data set. Hereby, it is important to emphasize two points in calculating $P_{\mathrm{d}}$ and $R^{2}$. First, we are comparing a mean-field theory to numerical simulations that contain 
the complexity of magnetization reversal in its complete detail. Second, we are using a finite size grid in our simulation while the analytic theory is derived for infinite systems. Naturally, the finite size will affect the $R^{2}$ and $P_{\mathrm{d}}$ calculation. Particularly, for data points near the beginning or end of the reversal curve, where only a few grains (hysterons) are reversed, the analytical theory for infinite hysteron numbers might not be accurate at all for the description of a finite system, independent from the validity of the mean-field approximation.

\section{B. Deviation From Redundancy}

As already mentioned above, fit quality measures such as $R^{2}$ are not necessarily foolproof because physical models and corresponding fit functions may be used in a regime, for which the underlying theory does not apply anymore. In such cases, data fits and extracted materials parameters might appear very accurate, while they are not. Thus, it would be a tremendous help, if an independent data set evaluation would be available that allows a separate measure of the suitability of the underlying theory. Specifically here, this evaluation should tell us how good an approximation the mean-field theory is for any given data set, so that we know to which confidence level we can rely on the $\Delta H(M, \Delta M)$ method.
We find that it is indeed possible to define such a quantity. To do so one has to recognize that within the meanfield approximation $\Delta H(M, \Delta M)$ data sets contain redundancy. The data set redundancy can be seen from Fig. 3. For illustration purposes, we pick six points: $(\mathrm{A}$ and $\mathrm{P}),(\mathrm{B}$ and $\mathrm{U}),(\mathrm{Q}$ and $\mathrm{V})$ located on the major loop, the $i$-th recoil curve and the $j$-th recoil curve, respectively. The $i$-th and $j$-th recoil curve start at $M_{\mathrm{rev}}=1-\Delta M_{i}$ and $1-\Delta M_{j}$, respectively. Without limiting the generality of this consideration, we furthermore assume that $\Delta M_{i}<\Delta M_{j}$ and require that the six points satisfy: $M_{\mathrm{A}}=M_{\mathrm{B}}=M, M_{\mathrm{P}}=M_{\mathrm{Q}}=M-\Delta M_{j}+\Delta M_{i}$ and $M_{\mathrm{U}}=M_{\mathrm{V}}=M-\Delta M_{j}$ where $M$ is an arbitrary value within $\left[-1,1-\Delta M_{i}\right]$. We then define

$$
\begin{aligned}
\Delta H_{i}(M) & =H_{\mathrm{B}}-H_{\mathrm{A}} \\
\Delta H_{j}\left(M-\Delta M_{j}+\Delta M_{i}\right) & =H_{\mathrm{Q}}-H_{\mathrm{P}} \\
\Delta H_{j}\left(M-\Delta M_{j}\right)-\Delta H_{i}\left(M-\Delta M_{j}\right) & =H_{\mathrm{V}}-H_{\mathrm{U}}
\end{aligned}
$$

Within the mean-field approximation, it is easy to prove that

$$
\left(H_{\mathrm{B}}-H_{\mathrm{A}}\right)+\left(H_{\mathrm{V}}-H_{\mathrm{U}}\right)-\left(H_{\mathrm{Q}}-H_{\mathrm{P}}\right)=0
$$

as shown in Appendix B. More generally, one finds that

$$
\Delta H_{i}(M)+\Delta H_{j}\left(M-\Delta M_{j}\right)-\Delta H_{i}\left(M-\Delta M_{j}\right)-\Delta H_{j}\left(M-\Delta M_{j}+\Delta M_{i}\right)=0
$$

This data set redundancy is due to the fact that successive recoil curves are not fully independent and contain repeated information. However, Eq. 13 is derived under the assumption of the mean-field approximation and is only true if the mean-field approximation is indeed fulfilled by the data set. For general data sets this property is not conserved. Therefore, we can define an $M$-dependent measure as

$$
r_{i j}(M)=\frac{\Delta H_{i}(M)+\Delta H_{j}\left(M-\Delta M_{j}\right)-\Delta H_{i}\left(M-\Delta M_{j}\right)-\Delta H_{j}\left(M-\Delta M_{j}+\Delta M_{i}\right)}{\Delta H_{i}(M)+\Delta H_{j}\left(M-\Delta M_{j}\right)+\Delta H_{i}\left(M-\Delta M_{j}\right)+\Delta H_{j}\left(M-\Delta M_{j}+\Delta M_{i}\right)}
$$

that monitors deviations from the mean-field approximation based upon the above redundancy criterion (Eq. 13). Eq. 14 has an $M$ definition range of $\left[\Delta M_{j}-1,1-\Delta M_{i}\right]$. For a general set of multiple recoil curves, the average deviation from redundancy measure can be defined as

$$
r=\frac{1}{n} \sum_{i, j}\left\langle r_{i j}^{2}(M)\right\rangle^{\frac{1}{2}}
$$

with $n$ being the total number of all the possible $(i, j)$ pairs ${ }^{17}$. Thus, $r$ is a quantitative measure that allows an accurate and independent check of how close or far any $\Delta H(M, \Delta M)$ data set is from fulfilling the mean-field approximation.
Note that in calculating $r$, we are comparing the data set with itself. Thus, the validity of the underlying meanfield approximation used for all the $\Delta H(M, \Delta M)$ data fits, can be assessed independently and from the data set alone.

An additional benefit in the calculation of $r$ is that the finite size inaccuracies at the definition range boundaries for the recoil curves will cancel out, at least to some degree. See Eq. 14. For $M=1-\Delta M_{i}$, the finite size inaccuracies in $\Delta H_{i}(M)$ and $\Delta H_{j}\left(M-\Delta M_{j}+\Delta M_{i}\right)$ will cancel out. Similarly, for $M=\Delta M_{j}-1$, the finite size inaccuracies in $\Delta H_{j}\left(M-\Delta M_{j}\right)$ and $\Delta H_{i}\left(M-\Delta M_{j}\right)$ will cancel out. In this sense, the deviation from redundancy measure is more robust than the fit quality measures. 

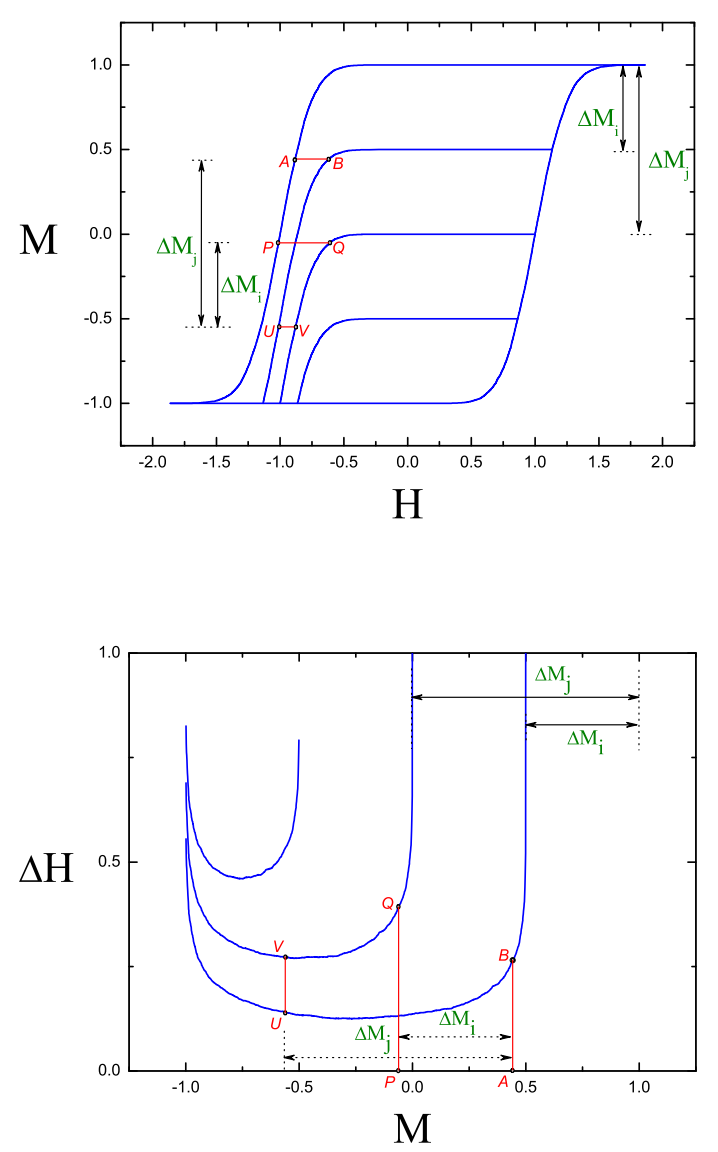

FIG. 3: (Top) The major hysteresis loop and three recoil curves. Throughout the paper, $M$ is normalized to its saturation value $M_{\mathrm{S}}$ and $H$ is normalized to the coercive field $H_{\mathrm{C}}$. The first two recoil curves start at $M_{\mathrm{rev}}=1-\Delta M_{i}$ and $1-\Delta M_{j}$, respectively, with $\Delta M_{i}<\Delta M_{j}$. For the six points $(\mathrm{A}$ and $\mathrm{P}),(\mathrm{B}$ and $\mathrm{U}),(\mathrm{Q}$ and $\mathrm{V})$ shown in the figure with $M_{\mathrm{A}}=M_{\mathrm{B}}=M, M_{\mathrm{P}}=M_{\mathrm{Q}}=M-\Delta M_{j}+\Delta M_{i}$ and $M_{\mathrm{U}}=M_{\mathrm{V}}=M-\Delta M_{j}$ where $M$ is an arbitrary value within $\left[-1,1-\Delta M_{i}\right]$, one can prove the existence of data redundancy, i.e. the equality $\left(H_{\mathrm{B}}-H_{\mathrm{A}}\right)+\left(H_{\mathrm{V}}-H_{\mathrm{U}}\right)=\left(H_{\mathrm{Q}}-H_{\mathrm{P}}\right)$ within the mean-field approximation. (Bottom) The corresponding $\Delta H(M, \Delta M)$ data for the three recoil curves.

\section{SIMULATION RESULTS}

To show that the $\Delta H(M, \Delta M)$ method fails reproducibly in a well-defined manner, we calculate $\Delta H(M, \Delta M)$ data sets for 4 different distributions.

For all these distributions, we tune the disorder parameter $\sigma$ from 1.6 to 1000 , but keep the ratio $h_{0} / \sigma$ to be a positive constant. This is done to avoid any negative switching fields, which would otherwise describe nonphysical behavior in violation of the second law of thermodynamics $\frac{18}{}$. For a Gaussian, $h_{0} / \sigma=5$ is generally big enough to avoid any negative $H_{\mathrm{S}}$ for system sizes of up to $1000^{2}$. For a Lorentzian, we choose $h_{0} / \sigma=5 \times 10^{3}$ for systems of size $100^{2}$ and $h_{0} / \sigma \sim 2 \times 10^{5}$ for systems of size $1000^{2}$. To avoid the long negative tails of the Lorentzian, we can truncate the distribution instead of choosing a huge $h_{0} / \sigma$ ratio, creating a new type of distribution, which we refer to as truncated Lorentzian distribution $D_{\mathrm{L}_{\mathrm{t}}}\left(H_{\mathrm{S}}\right)$ in the following. Further details, including the definition of $\sigma$, for this distribution are described in Appendix C. For this $D_{\mathrm{L}_{\mathrm{t}}}\left(H_{\mathrm{S}}\right)$ distribution, we also choose $h_{0} / \sigma=5$ in our calculations. For the Lognormal distribution, there is by definition no distribution density for negative fields. But we still choose $h_{0} / \sigma=5$ to make it comparable with the Gaussian and the truncated Lorentzian distribution.

\section{A. Comparison with the mean-field approximation}

Key results of our numerical hysteresis loop calculations for all these different switching field distributions are shown in Figs. 4 7 . In each case, we show plots for $\sigma=1.6,5$ and 50 only to illustrate the general trends. All the calculations shown here are done in $D=2$ dimensions for linear system size $L=1000$, i.e. $L^{2}=10^{6}$ hysterons.

At first, we discuss the results for the Gaussian $D\left(H_{\mathrm{S}}\right)$ distribution in detail. Fig. (4 displays the results for different $\sigma$ 's in different rows: (Top) $\sigma=1.6$. (Middle) $\sigma=5$. (Bottom) $\sigma=50$. For each $\sigma$, we calculate a complete set of $M(H)$ curves, both the saturation hysteresis loop and recoil curves, as shown in the left column of Fig. 4 Note that here and throughout the paper, $M$ (or $\Delta M$ ) is normalized to the saturation value $M_{\mathrm{S}}$ and $H$ (or $\Delta H$ ) is normalized to the coercive field $H_{\mathrm{C}}$. In particular, we choose 5 equally-spaced recoil curves, for which the distance to saturation is given by $\Delta M_{i}=i / 3$. From the left column of Fig. 4, one can see that the hysteresis curves get broader for higher $\sigma$. Note that it is not the larger $\sigma$ itself that causes this effect, because this type of broadening is taken out due to the normalization of $H$ with $H_{\mathrm{C}}$, and the constant $h_{0} / \sigma$ ratio $\frac{19}{}$. The difference in shape here actually reflects the fact that for lower $\sigma$, one gets correlated magnetization reversal which sharpens the macroscopic switching field distribution substantially.

In the middle column of Fig. 4, we show the corresponding $\Delta H(M, \Delta M)$ curves (solid lines) derived from the simulated $M(H)$ curves, as well as the mean-field approximation of the $\Delta H(M, \Delta M)$ curves (dotted lines) calculated from Eq. 5a, The mean-field curves are calculated by using the exact input parameter and are not a least square fit. This allows for a clear illustration of the deviations from mean-field behavior. From Fig. 4(d), we see that for small $\sigma$ (corresponding to strong hysteron interactions) the difference between the numerical result and the mean-field approximation is large. For intermediate $\sigma$ (corresponding to intermediate hysteron interactions), the difference diminishes but is still visi- 
ble, especially near the negative saturation $M=-1$, as shown in Fig. 4(e). For high $\sigma$ (corresponding to weak hysteron interactions), the difference is so small that it is not visible in Fig. U(f). It should be mentioned that due to the constant $h_{0} / \sigma$ ratio and the normalization of $\Delta H$, the mean-field $\Delta H(M, \Delta M)$ curves look almost identical for different $\sigma$ 's $\frac{19}{}$. Furthermore, it is apparent that the $\Delta H(M, \Delta M)$ curves obtained from numerical simulations are asymmetric, in particular for small $\sigma$. They show much larger deviations from the mean-field approximation on the left hand side, i.e. near negative saturation $M=-1$. This can also be seen in the hysteresis loops themselves, where the curves seem to bundle up near negative saturation for small $\sigma$ values.

In the right column of Fig. 4, we show numerical values for the deviation from redundancy measures $r_{i j}(M)$ which are calculated from the simulated recoil curves shown in the left column. Due to the definition range of $r_{i j}(M)$, only the recoil curve pairs $(i, j)=(1,2),(1,3)$, $(1,4)$ and $(2,3)$ produce data. From Fig. $4(\mathrm{~g})$, we see that for small $\sigma$ the deviation from data redundancy is quite substantial for the whole $M$ definition range and for all the recoil curve pairs. For intermediate $\sigma$, the deviation becomes smaller but is still visible, as shown in Fig. 近(h). For high $\sigma$, the deviation is almost negligible in the whole $M$ definition range and for all recoil curve pairs, as one can see from Fig. $4(i)$. Small non-vanishing values occurring at the boundaries of the $M$ definition range are just due to the incompletely canceled finite size inaccuracies, as discussed in Sec. IVB

For the other $D\left(H_{\mathrm{S}}\right)$ distributions, we observe very similar results as shown in Figs. 5 - 7 . Thus, one has to realize that for small $\sigma$, i.e. strong hysteron interactions, the $\Delta H(M, \Delta M)$ method is not accurate. The meanfield approximation doesn't match the numerical result and deviations from redundancy are large. This is the expected result because once coupling dominates the magnetization reversal the mean-field approximation will not be valid any more. On the other hand, for large $\sigma$, i.e. weak hysteron interactions, the $\Delta H(M, \Delta M)$ method works very well, which is indicated by both the small deviation from redundancy and the match of the meanfield approximation to the numerical results.

\section{B. Emergent feature of the $\Delta H(M, \Delta M)$ method}

The similarities in the failure of the $\Delta H(M, \Delta M)$ method for different $D\left(H_{\mathrm{S}}\right)$ distributions indicates that this method may be not very sensitive to the particular type of distribution in general. To study this further, we plot the reliability measures against the tuning parameter $\sigma$ for all $D\left(H_{\mathrm{S}}\right)$ distributions in Fig. 8 .

The fit quality measures $P_{\mathrm{d}}$ and $R^{2}$ are shown in Fig. $8(a)$ and (b), respectively. We see that $P_{\mathrm{d}}$ approaches 0 with increasing $\sigma$, which means that for high $\sigma$, the input value of $\sigma$ can be recovered with very high accuracy by fitting the $\Delta H(M, \Delta M)$ data to the mean-field fit function. In other words, the $\Delta H(M, \Delta M)$ method works well for high $\sigma$. Furthermore, we see that with increasing $\sigma, R^{2}$ approaches 1 corroborating a successful fit of the $\Delta H(M, \Delta M)$ data in this regime. The average deviation from redundancy $(r)$ is shown in Fig. 8(c). It is clearly seen that with increasing $\sigma, r$ approaches 0 , i.e. data redundancy is obtained, which is the key feature of the mean-field approximation.

The reliability range of the $\Delta H(M, \Delta M)$ method can be obtained from the reliability measures shown in Fig. 8. We find that for all the four $D\left(H_{\mathrm{S}}\right)$ distributions, with the definitions of $\sigma$ given in Sec. II the $\Delta H(M, \Delta M)$ method works virtually perfect for $\sigma \geq \sigma_{0}$ with $\sigma_{0}$ being approximately equal to 20 . Here, $\sigma_{0}$ is just a rough criterion, above which the reliability measures have merged into their mean-field approximation values. It should be emphasized that the $\sigma_{0}$ values obtained from all three reliability measures are fairly consistent. If there are differences at all, $r$ appears to show the highest sensitivity to deviations from the mean-field approximation, while $R^{2}$ seems to be slightly less sensitive. This is important, because $r$ can be evaluated without any fit from a data set alone. So, the independent reliability test is the most sensitive measure and gives one confidence that not only the $\Delta H(M, \Delta M)$ method fails in a well-defined way, but also that one can very reliably check for this failure mode.

Finally, we note that the different $r$ vs. $\sigma$ curves in Fig. 8(c) for the different distribution types track each other almost exactly. However, this particular observation dependents somewhat on how exactly we define $\sigma$ in the various $D\left(H_{\mathrm{S}}\right)$ distributions, because only for the Gaussian and Lognormal distribution are we using the natural definition given by the standard deviation. Thus, the curve collapse seen in Fig. 8(c) might be partially artificial. $P_{\mathrm{d}}$ and $R^{2}$ on the other hand do not exhibit such a collapse, not even for the Gaussian and Lognormal distributions as is apparent from Fig. 8(a) and (b). The fact that the two kinds of reliability measures show different behavior can be understood in the following way. As mentioned in the end of Sec. IVA and Sec. IVB finite size inaccuracies at the definition range boundaries of the recoil curves will not affect the calculation of $r$ very much due to the cancellation effect. But it will affect the calculation of the fit quality measures, both $P_{\mathrm{d}}$ and $R^{2}$. Generally speaking, the shape of the hysteresis loops and recoil curves depends on the particular type of the chosen $D\left(H_{\mathrm{S}}\right)$. Consequently, the finite size inaccuracies will also depend on $D\left(H_{\mathrm{S}}\right)$. As a result, we see different (similar) behaviors of the fit quality measures (deviation from redundancy measure) for different $D\left(H_{\mathrm{S}}\right)$ distributions at small $\sigma$. In this sense, it is natural to choose the deviation from redundancy measure $r$ as the best measure to determine the reliability range of the $\Delta H(M, \Delta M)$ method. 

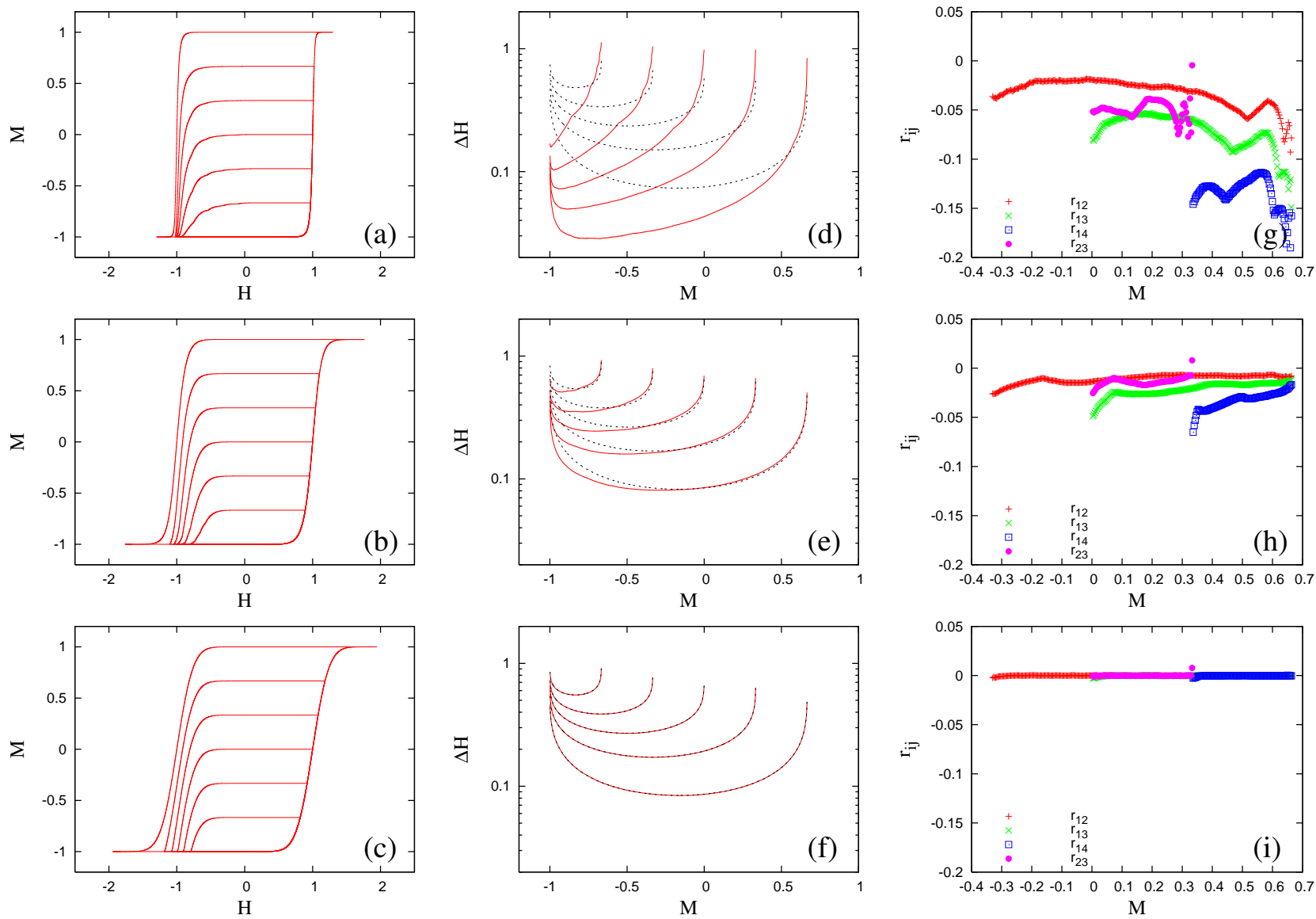

FIG. 4: Numerical results using a Gaussian distribution RSFHM. Rows: (Top, fig.a,d,g): $\sigma=1.6$. (Middle, fig.b.e.h): $\sigma=5$. (Bottom, fig.c,f,i): $\sigma=50$. Columns: (Left, fig.a,b,c): $M(H)$ curves, main loop and 5 recoil curves. (Middle, fig.d,e,f): $\Delta H(M, \Delta M)$ curves for the 5 recoil curves: (solid lines) numerical result; (dotted lines) mean-field approximation. (Right, fig.g,h,i): $M$-dependent deviation from redundancy $\left(r_{i j}(M)\right)$ for all the possible recoil curve pairs.

\section{SUMMARY}

We study the $\Delta H(M, \Delta M)$ method and its reliability by means of numerical simulations of the zerotemperature random switching field hysteron model. We present strong evidence that the $\Delta H(M, \Delta M)$ method, which is based on the mean-field approximation, has a well-defined reliability range. This reliability range can be checked with two types of independent measures: deviation from redundancy and fit quality. The former is the superior tool because it is calculated from the data set alone and is independent from any inaccuracies that might be induced by data fitting procedures itself.

\section{ACKNOWLEDGMENT}

We thank M. Delgado for valuable discussions. Work done at UIUC was conducted on the Beowolf cluster of the Materials Computation Center at UIUC and acknowledges the support of NSF Grant No. DMR 0314279 and NSF Grant No. DMR 03-25939 ITR (Materials Com- putation Center).

\section{APPENDIX A: MAPPING RSFHM TO RFIM}

For the zero-temperature non-equilibrium RFIM, a local metastable dynamics has been introduced by Sethna et al. ${ }^{14}$ to study the disorder-induced phase transition in the hysteretic behavior at $T=0$ : Initially, all spins point DOWN, as $H$ is slowly increased from $-\infty$ to $\infty$ and decreased back to $-\infty$, each spin flips deterministically when its effective local field

$$
h_{i}^{\mathrm{eff}}=J \sum_{j} s_{j}+H+h_{i}
$$

changes sign. For the zero-temperature non-equilibrium RSFHM, we introduce a similar local metastable dynamics: Initially, all spins point DOWN, as $H$ is slowly increased from $-\infty$ to $\infty$ and decreased back to $-\infty$, each hysteron flips deterministically when its effective local 

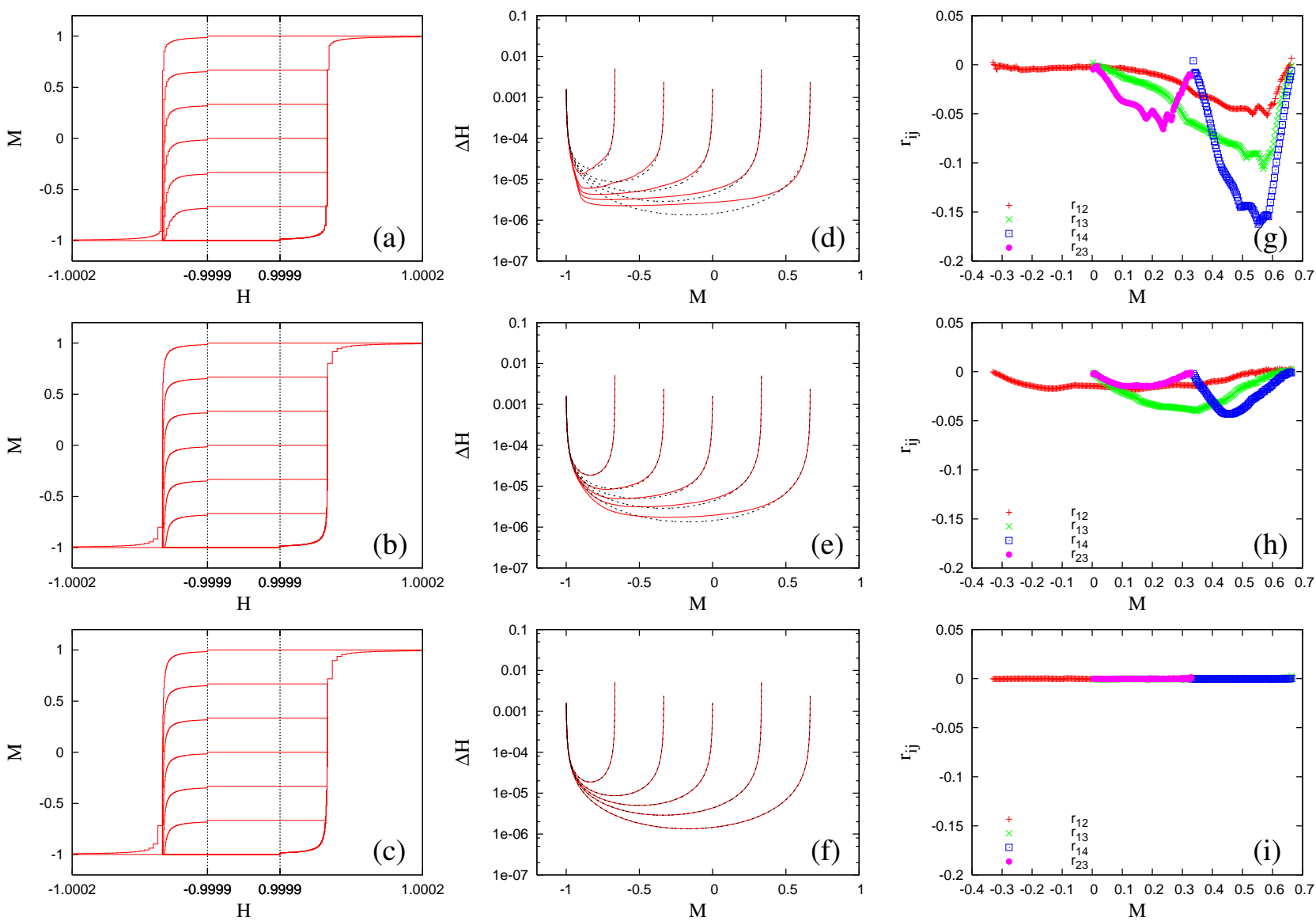

FIG. 5: Numerical results using a Lorentzian distribution RSFHM. Rows: (Top, fig.a,d,g): $\sigma=1.6$. (Middle, fig.b.e.h): $\sigma=5$. (Bottom, fig.c,f,i): $\sigma=50$. Columns: (Left, fig.a,b,c): $M(H)$ curves, main loop and 5 recoil curves. Note that due to the very large $h_{0} / \sigma$ ratio $\left(\sim 2 \times 10^{5}\right.$ for this system size $1000^{2}$, chosen to avoid negative $\left.H_{\mathrm{S}}\right)$, the differences between the major loop and all the recoil curves are extremely hard to see from the $M(H)$ plot itself. The small differences will be more clear with $\log$ scale as shown in the middle column. (Middle, fig.d,e,f): $\Delta H(M, \Delta M)$ curves for the 5 recoil curves: (solid lines) numerical result; (dotted lines) mean-field approximation. Here, we see that normalized $\Delta H$ values are very small compared to the Gaussian $D\left(H_{\mathrm{S}}\right)$ case due to the large $h_{0} / \sigma$ ratio. (Right, fig.g,h,i): $M$-dependent deviation from redundancy $\left(r_{i j}(M)\right.$ ) for all the possible recoil curve pairs.

field

$$
H_{i}^{\mathrm{eff}}=J \sum_{j} S_{j}+H+\operatorname{sgn}\left(S_{i}\right) H_{\mathrm{S} i}
$$

changes sign.

Considering the only slight difference between the RFIM and the RSFHM, it is possible to introduce a simple mapping: $h_{i} \leftrightarrow \operatorname{sgn}\left(S_{i}\right) H_{\mathrm{S} i}$ with $H_{\mathrm{S} i}>0$. This enables us to calculate the $M(H)$ curve of the interacting hysterons by means of simple software adaptation.

\section{APPENDIX B: PROOF OF DATA REDUNDANCY IN MEAN-FIELD THEORY}

As shown in Fig. 3, we choose six states (A, B, P, Q, $\mathrm{U}$ and $\mathrm{V}$ ) from the major loop and recoil curves with

$$
\begin{aligned}
& M_{\mathrm{A}}=M_{\mathrm{B}}=M \\
& M_{\mathrm{P}}=M_{\mathrm{Q}}=M-\Delta M_{j}+\Delta M_{i} \\
& M_{\mathrm{U}}=M_{\mathrm{V}}=M-\Delta M_{j}
\end{aligned}
$$

and $M$ is an arbitrary value within $\left[-1,1-\Delta M_{i}\right]$. The hysteron distributions for the six states are shown in Fig. 9. Shadowed areas denote DOWN-hysterons $\left(S_{i}=\right.$ $-1)$, while open areas indicate UP-hysterons $\left(S_{i}=+1\right)$. In the following, we use the symbol $n_{\downarrow}$ and $n_{\uparrow}$ for the density of DOWN-hysteron and UP-hysteron, respectively. For the states chosen from the upper branch of the major loop with magnetization $M_{0}$, it is easy to get $n_{\downarrow}=\left(1-M_{0}\right) / 2$. For example,

$$
\begin{aligned}
& n_{\downarrow}(A)=(1-M) / 2 \\
& n_{\downarrow}(P)=\left(1-M-\Delta M_{i}+\Delta M_{j}\right) / 2
\end{aligned}
$$



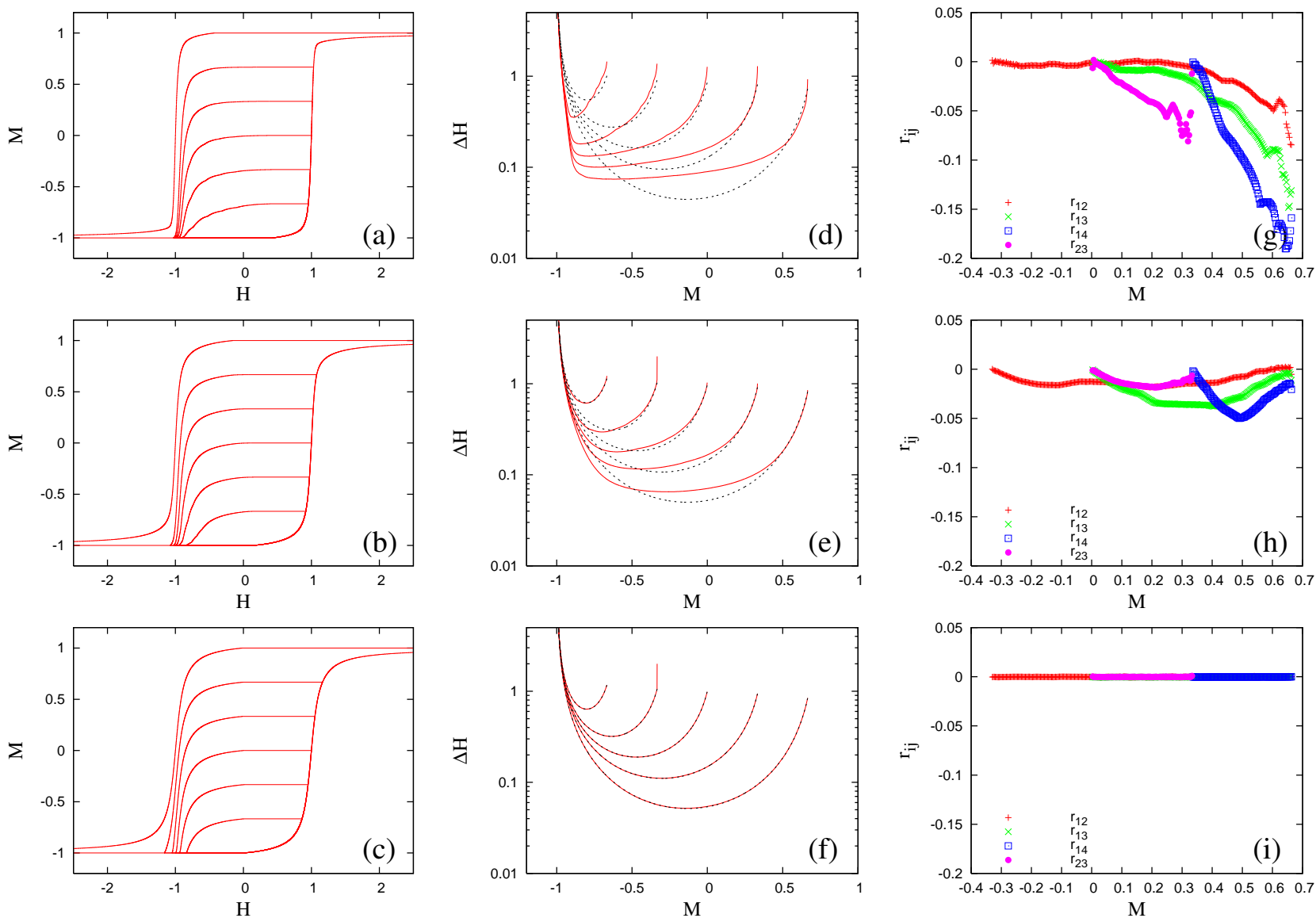

FIG. 6: Numerical results using a truncated Lorentzian distribution RSFHM. Rows: (Top, fig.a,d,g): $\sigma=1.6$. (Middle, fig.b.e.h): $\sigma=5$. (Bottom, fig.c,f,i): $\sigma=50$. Columns: (Left, fig.a,b,c): $M(H)$ curves, main loop and 5 recoil curves. (Middle, fig.d,e,f): $\Delta H(M, \Delta M)$ curves for the 5 recoil curves: (solid lines) numerical result; (dotted lines) mean-field approximation. (Right, fig.g,h,i): $M$-dependent deviation from redundancy $\left(r_{i j}(M)\right)$ for all the possible recoil curve pairs.

For states chosen from the recoil curves with magnetization $M_{0}$, there are two shadowed areas, which can be denoted as $n_{\downarrow 1}$ and $n_{\downarrow 2}$. Note that the left one $n_{\downarrow 1}$ is just due to the distance from saturation at the starting point of the recoil curve, i.e. $\Delta M$, so that $n_{\downarrow 1}=\Delta M / 2$. For example, $\mathrm{B}$ and $\mathrm{U}$ are picked from the $i$-th recoil curve with distance from saturation $\Delta M_{i}$, so that

$$
n_{\downarrow 1}(B)=n_{\downarrow 1}(U)=\Delta M_{i} / 2
$$

Similarly, for Q and V, we have

$$
n_{\downarrow 1}(Q)=n_{\downarrow 1}(V)=\Delta M_{j} / 2
$$

Combining this with the number conservation equation $n_{\downarrow}=n_{\downarrow 1}+n_{\downarrow 2}=\left(1-M_{0}\right) / 2$, we find

$$
\begin{aligned}
& n_{\downarrow 2}(B)=\left(1-M-\Delta M_{i}\right) / 2 \\
& n_{\downarrow 2}(Q)=\left(1-M-\Delta M_{i}\right) / 2 \\
& n_{\downarrow 2}(U)=\left(1-M-\Delta M_{i}+\Delta M_{j}\right) / 2 \\
& n_{\downarrow 2}(V)=(1-M) / 2
\end{aligned}
$$

It follows that

$$
\begin{aligned}
n_{\downarrow}(A) & =n_{\downarrow 2}(V) \\
n_{\downarrow}(P) & =n_{\downarrow 2}(U) \\
n_{\downarrow 2}(B) & =n_{\downarrow 2}(Q)
\end{aligned}
$$



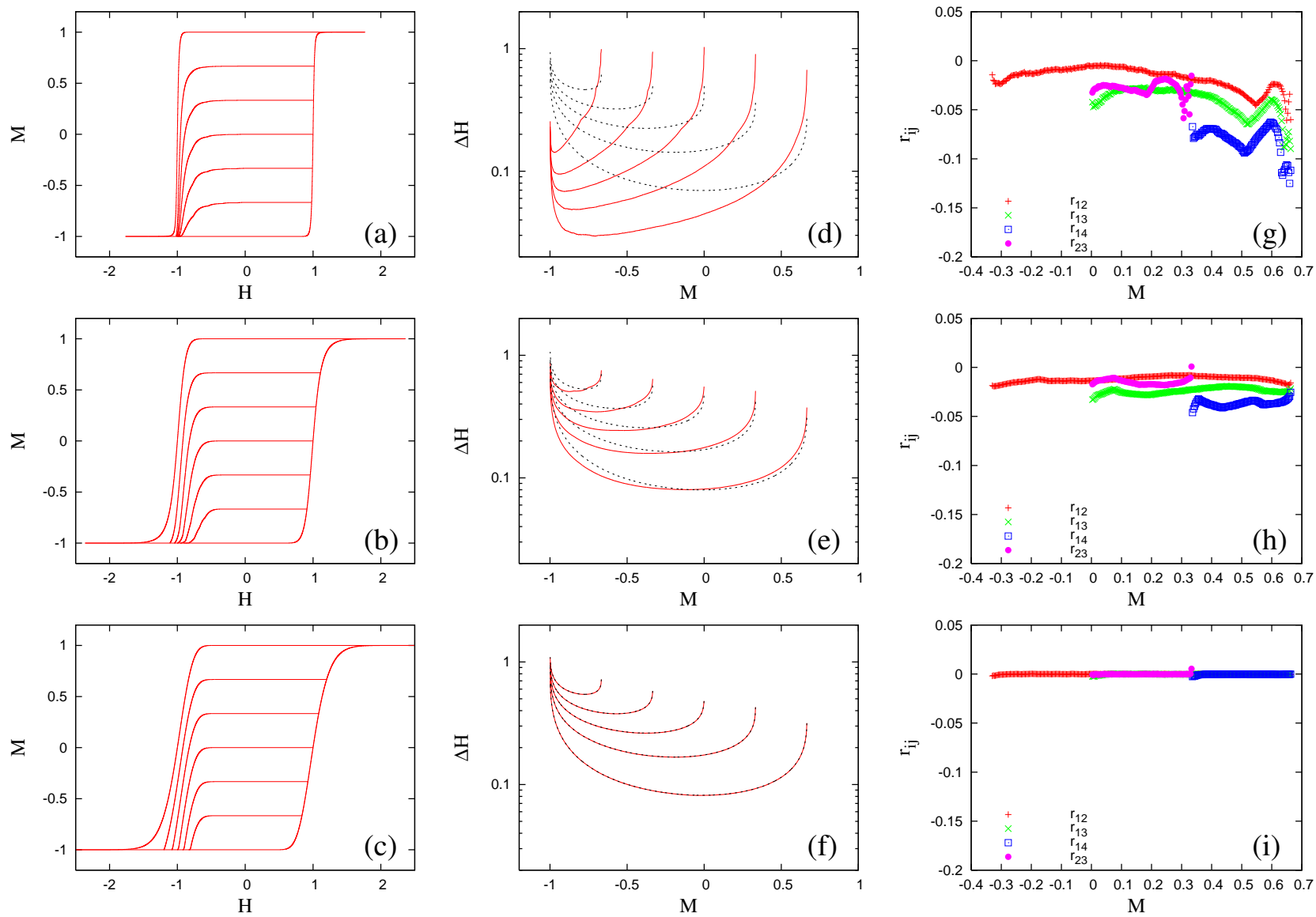

FIG. 7: Numerical results using a Lognormal distribution RSFHM. Rows: (Top, fig.a,d,g): $\sigma=1.6$. (Middle, fig.b.e.h): $\sigma=5$. (Bottom, fig.c,f,i): $\sigma=50$. Columns: (Left, fig.a,b,c): $M(H)$ curves, main loop and 5 recoil curves. (Middle, fig.d,e,f): $\Delta H(M, \Delta M)$ curves for the 5 recoil curves: (solid lines) numerical result; (dotted lines) mean-field approximation. (Right, fig.g,h,i): $M$-dependent deviation from redundancy $\left(r_{i j}(M)\right)$ for all the possible recoil curve pairs.

$$
\begin{aligned}
H_{\mathrm{A}}+H_{\mathrm{int}}(M) & =H_{\mathrm{V}}+H_{\mathrm{int}}\left(M-\Delta M_{j}\right) \\
H_{\mathrm{P}}+H_{\mathrm{int}}\left(M-\Delta M_{j}+\Delta M_{i}\right) & =H_{\mathrm{U}}+H_{\mathrm{int}}\left(M-\Delta M_{j}\right) \\
H_{\mathrm{B}}+H_{\mathrm{int}}(M) & =H_{\mathrm{Q}}+H_{\mathrm{int}}\left(M-\Delta M_{j}+\Delta M_{i}\right)
\end{aligned}
$$

So, overall we find

$$
\left(H_{\mathrm{B}}-H_{\mathrm{A}}\right)+\left(H_{\mathrm{V}}-H_{\mathrm{U}}\right)-\left(H_{\mathrm{Q}}-H_{\mathrm{P}}\right)=0
$$

Q.E.D

\section{APPENDIX C: $\Delta(H, \Delta M)$ FORMULA FOR THE TRUNCATED LORENTZIAN DISTRIBUTION}

In numerical simulation, the random switching fields with any distributions are generated by a random number generator. To avoid any negative tails in the Lorentzian distribution, we can artificially suppress any negative random numbers and instead create another random number for the switching field until we get a positive one. The corresponding switching field distribution is then represented by the truncated Lorentzian distribution $D_{\mathrm{L}_{\mathrm{t}}}\left(H_{\mathrm{S}}\right)$ :

$$
D_{\mathrm{L}_{\mathrm{t}}}\left(H_{\mathrm{S}}\right)= \begin{cases}0 & \text { for } H_{\mathrm{S}}<0 \\ \frac{2 w}{\pi} \frac{C}{w^{2}+4\left(H_{\mathrm{S}}-h_{0}\right)^{2}} & \text { for } H_{\mathrm{S}} \geq 0\end{cases}
$$

with $C$ given by the condition that 

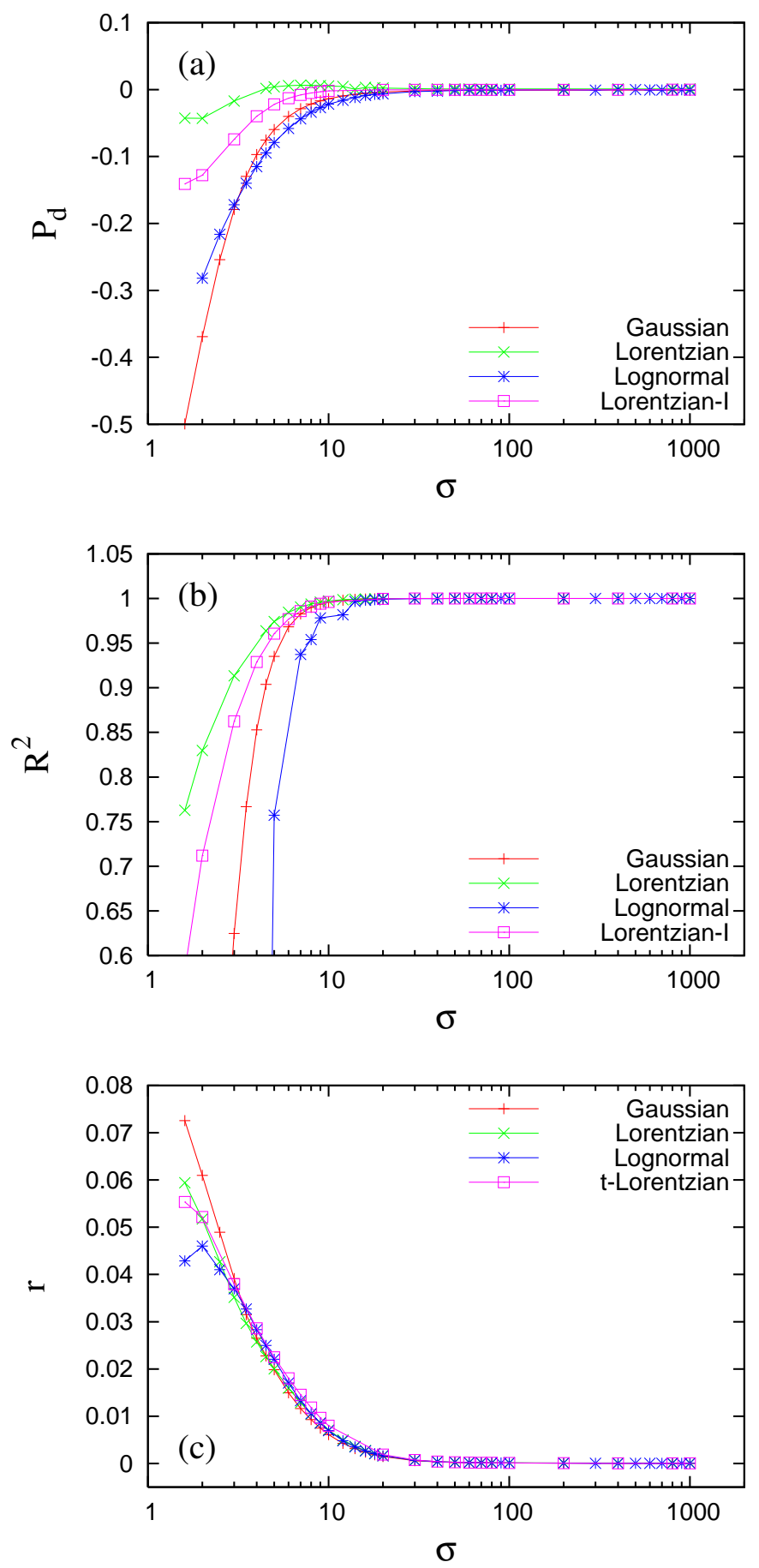

For the truncated Lorentzian distribution, we define the disorder parameter $\sigma=w$ to make it comparable with the Lorentzian distribution.

The integral function is given by:

$$
\begin{aligned}
I_{\mathrm{L}_{\mathrm{t}}}(x) & =\int_{-\infty}^{x} D_{\mathrm{L}_{\mathrm{t}}}\left(H_{\mathrm{S}}\right) \mathrm{d} H_{\mathrm{S}} \\
& =\frac{C}{\pi}\left[\tan ^{-1}\left(\frac{2\left(x-h_{0}\right)}{w}\right)+\theta\right]
\end{aligned}
$$

so that

$$
I_{\mathrm{L}_{\mathrm{t}}}^{-1}(y)=h_{0}+\frac{w}{2} \tan \left(\frac{\pi}{C} y-\theta\right)
$$

From this result, we derive

FIG. 8: Reliability measures for the different $D\left(H_{\mathrm{S}}\right)$ : (a) $P_{\mathrm{d}}$. (b) $R^{2}$. (c) $r$.

$\int_{-\infty}^{+\infty} D_{\mathrm{L}_{\mathrm{t}}}\left(H_{\mathrm{S}}\right) \mathrm{d} H_{\mathrm{S}}=1$. Specifically, one finds that

$$
C=\left(\frac{1}{2}+\frac{\theta}{\pi}\right)^{-1}
$$

with $\theta=\tan ^{-1}\left(\frac{2 h_{0}}{w}\right)$.

$$
\begin{aligned}
\Delta H_{\mathrm{L}_{\mathrm{t}}}(M, \Delta M)= & \frac{w}{2}\left\{\tan \left[\left(\frac{\pi}{2}+\theta\right)\left(\frac{1-M}{2}\right)-\theta\right]\right. \\
& \left.-\tan \left[\left(\frac{\pi}{2}+\theta\right)\left(\frac{1-M-\Delta M}{2}\right)-\theta\right]\right\}
\end{aligned}
$$

as the analytic mean-field solution. We notice that due to the truncation induced asymmetry in the distribution itself, $\Delta H_{\mathrm{L}_{\mathrm{t}}}(M, \Delta M)$ depends on both $w$ and $h_{0}$. 
1 M. L. Plumer, J. van Ek, and D. Weller, eds., The Physics of Ultrahigh-Density Magnetic Recording (Springer Series in Surface Sciences) (Springer, 2001).

2 Y. Shimizu and H. N. Bertram, IEEE Trans. Mag 39, 1846 (2003).

${ }^{3}$ I. Tagawa and Y. Nakamura, IEEE Trans. Mag 27, 4975 (1991).

4 C. R. Pike, A. P. Roberts, and K. L. Verosub, J. Appl. Phys. 85, 6660 (1999).

${ }^{5}$ R. J. M. van de Veerdonk, X. Wu, and D. Weller, IEEE Trans. Mag 38, 2450 (2002).

${ }^{6}$ R. J. M. van de Veerdonk, X. Wu, and D. Weller, IEEE Trans. Mag 39, 590 (2003).

7 A. Berger, Y. H. Xu, B. Lengsfield, Y. Ikeda, and E. E. Fullerton, IEEE Trans. Mag 41, 3178 (2005).

8 A. Berger, B. Lengsfield, and Y. Ikeda, J. Appl. Phys. 99, $08 \mathrm{E} 705$ (2006).

9 M. Winklhofer and G. T. Zimanyi, J. Appl. Phys. 99, 08E710 (2006).

10 I. D. Mayergoyz, Phys. Rev. Lett. 56, 1518 (1986).

11 N. Goldenfeld, Lectures on Phase Transitions and the Renormalization Group (Westview Press, 2005).

12 K.-Z. Gao and H. N. Bertram, J. Appl. Phys. 96, 7753 (2004).
13 T. Shimatsu, T. Kondo, K. Mitsuzuka, S. Watanabe, H. Aoi, H. Muraoka, and Y. Nakamura, IEEE Trans. Mag 42, 2384 (2006).

14 J. P. Sethna, K. A. Dahmen, S. Kartha, J. A. Krumhansl, B. W. Roberts, and J. D. Shore, Phys. Rev. Lett. 70, 3347 (1993).

15 M. C. Kuntz, O. Perkovic, K. A. Dahmen, B. W. Roberts and J. P. Sethna, Comp. Sci. Eng. 1, 73 (1999).

16 N. R. Draper and H. Smith, Applied regression analysis (Wiley, New York, 1966).

17 Since the definition range of $M$ in $r_{i j}(M)$ is $\left[\Delta M_{j}-1,1-\right.$ $\left.\Delta M_{i}\right]$, we must have $1-\Delta M_{i}>\Delta M_{j}-1$, i.e. $\Delta M_{i}+$ $\Delta M_{j}<2$.

18 L. D. Landau and E. M. Lifshitz, Statistical Physics Part 1 (Butterworth Heinemann, 3rd edition, 1984).

19 This statement only makes sense if $H_{\mathrm{C}}$ and $h_{0}$ are very similar, which should be fulfilled as long as the hysteron coupling is not too strong and the main cause of hysteresis is the single hysteron hysteresis. In fact, in our simulations, we find that $h_{0}$ and $H_{\mathrm{C}}$ are almost identical (or very similar) for high $\sigma$. Moreover, one could make this an exact statement by normalizing $H$ and $\Delta H$ to $h_{0}$. 